\title{
Development of lightweight optical segments for adaptive optics
}

\author{
M. Ghigo ${ }^{(1)}$, R. Canestrari ${ }^{(1,2)}$, S. Basso ${ }^{(1)}$, D. Spiga ${ }^{(1)}$ \\ (1) INAF-Osservatorio Astronomico di Brera - Via Bianchi 4623807 Merate (Lc) Italy \\ ${ }^{(2)}$ Università degli Studi dell'Insubria - Via Valleggio 1122100 Como (Co) Italy \\ Email: mauro.ghigo@brera.inaf.it rodolfo.canestrari@brera.inaf.it stefano.basso@brera.inaf.it \\ daniele.spiga@brera.inaf.it
}

\begin{abstract}
The large telescopes nowadays under development will have the adaptive optics systems fully integrated from the beginning of the project. These optics are in fact an essential component that is necessary for the full exploitation of the performances obtainable from the large optics foreseen in these instruments. Due to the large reflecting areas of these telescopes their adaptive optics systems will use probably thin segmented mirrors, assembled to create a single surface, placed along the optical train. Today, a number of telescopes (MMT, LBT, etc) have the monolithic secondary mirror of the instrument used as a component of the adaptive optic system. The technique used for the production of these single pieces thin mirror shells, typically having convex shape, is not well suited for the manufacturing of the large number of segments necessaries for the future telescopes. Infact, the procedure foresees the thinning of conventional thick mirrors, a technique expensive and time consuming. It is hence necessary to find a better approach able to produce thin optical segments in a cost effective way and with short delivery time. In this study, financed in the frame of OPTICON-FP6, the Astronomical Observatory of Brera (INAF-OAB) is investigating a technique for the manufacturing of these optical components that has the potential to fulfill these requirements. The curved optical segments that are under development will have a thickness of few $\mathrm{mm}$ and will be made in Borofloat ${ }^{\mathrm{TM}}$ glass. The technique foresees the thermal slumping of thin glass segments using a high quality ceramic mold as a master to impart a precise shape to the glass. The initially flat glass segment is placed onto the mold and then, by means of a suitable thermal cycle, the material is softened so to copy the master shape. If necessary, at the end of the slumping is foreseen the correction of the eventual remaining errors using the Ion Beam Figuring technique. This paper describes the process of production of the optical segments and the status of the investigation.
\end{abstract}

Keywords: Segmented Optics, Glass Slumping, Ion Beam Figuring, E-ELT

\section{INTRODUCTION}

The adaptive optics systems foreseen for the next generation of telescopes, having diameters in the range of 20-40 meters, will be probably segmented because they will have dimensions much larger than those that nowadays are installed in the present instruments. For example the secondary adaptive mirror of the Large Binocular Telescope ${ }^{(1,2)}$ (LBT) has a diameter of $91 \mathrm{~cm}$ and thickness $1.6 \mathrm{~mm}$, while the Multi Mirror Telescope ${ }^{(3)}$ (MMT) has an adaptive secondary with size of $64 \mathrm{~cm}$ and thickness of $2 \mathrm{~mm}$. Adaptive surfaces in glass with these dimensions can be manufactured using a technique that foresees the thinning of conventional thick glass blanks. This process is very expensive, delicate and time consuming. As visible in Fig. 1, the first step of the process uses a thick glass meniscus. Its surfaces are grinded until they have the same radius of curvature of a stiff support that will act as a blocking-body. In the next step of the procedure the meniscus and the support are glued together by means of a very thin layer of pitch. When the two pieces are glued it is then possible to continue the grinding of the upper surface of the meniscus and to reach the desired thickness, typically in the range of $1-2 \mathrm{~mm}$. At the end of the grinding and figuring of the surface it is then possible to detach the two pieces softening the pitch by heating. The convex thinned meniscus obtained in this way is then used as reflective surface of the adaptive optic. It is clear that a procedure of this type is useful for the production of single pieces but it is not well suited for the production of the large number of segments necessaries for the assembly of a large segmented adaptive optic. In this case it could be better to follow a different approach, able to produce the optical surfaces by means of a replica process. A possible technique using this philosophy is the molding of thin glass sheets so to impart them the precise shape of a master. This technique is generally referred as "slumping" because the glass sheet is placed above a mould having the complementary shape to be produced and then a suitable thermal cycle is applied.

Astronomical Adaptive Optics Systems and Applications III, edited by Robert K. Tyson, Michael Lloyd-Hart Proc. of SPIE Vol. 6691, 66910K, (2007) · 0277-786X/07/\$18 - doi: 10.1117/12.734439 
When sufficiently heated the glass will soften enough to slump onto the mould surface and adapt to its shape. In the end of the procedure, after the cooling down of the glass and mould, the glass sheet will be separated from the mould. At his point the surface of the glass sheet will have the requested optical shape and will be (ideally) ready to be coated with a reflective layer and used as an optical thin shell in an adaptive system. The advantage of this approach is that, once the mould has been manufactured, it is possible to replicate very quickly a number of segments with a low cost of production.

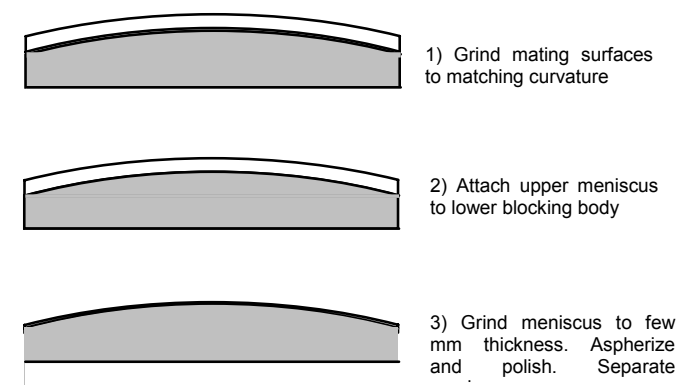

Fig. 1 Present manufacturing technique for Thin Mirrors Shell

The INAF-Astronomical Observatory of Brera (INAF-OAB) is developing a technique for the slumping of thin glass optical segments $^{(4)}$ for adaptive optics under a study financed in the frame of OPTICON FP6. This investigation is part of a larger effort dedicated to the development of the European Extremely Large Telescope (E-ELT) that will have a segmented main mirror with a diameter of 42 meters. The aim of this study is to validate the slumping technique for the manufacturing of adaptive optics with the production of a concave surface spherical demonstrator in Borofloat ${ }^{\mathrm{TM}}$ glass, having a diameter of $50 \mathrm{~cm}$, radius of about 4 meters and thickness of $1.6 \mathrm{~mm}$. This thin glass shell demonstrator, once manufactured, will be placed on an adaptive support that is under development in the INAF-Astronomical Observatory of Arcetri (INAF-Arcetri) that is partner with INAF-OAB in this development. The dimensions of the real segments that could be manufactured with this technique, at this time has been set at about $1.5 \mathrm{~m}$, as required in the case of a segmented secondary mirror for E-ELT. The proposed approach for the production of the slumped segments is shown in Fig. 2 and consists in the following steps: a mould having the complementary shape desired for the optical segment is manufactured in a suitable material that has a CTE as similar as possible to that of the glass to be slumped. The microroughness surface finishing of the mould will be similar to that necessary for the slumped segment. This requirement is necessary because the procedure foresees to copy not only the geometry of the mould but also its higher spatial frequencies and in particular a part of its microroughness.

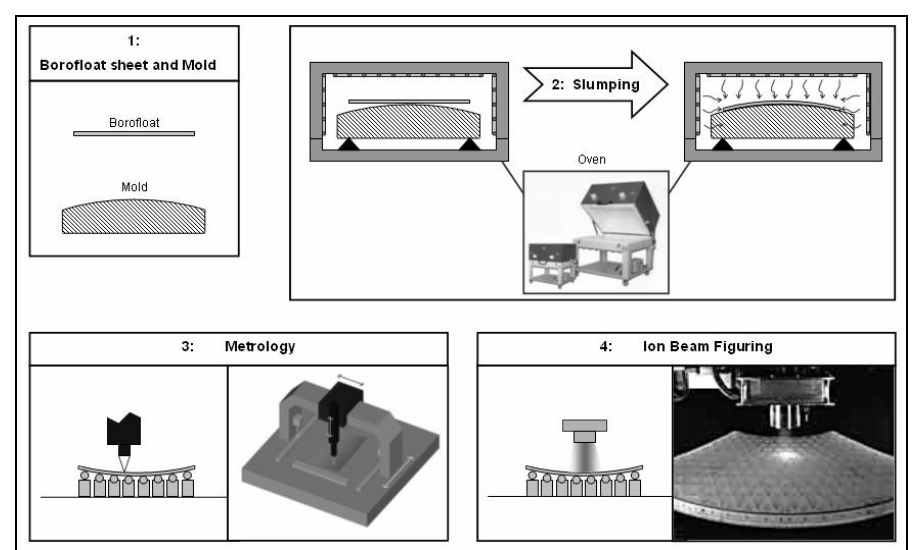

Fig. 2 Slumping technique for manufacturing Thin Mirrors Shell

After a deep cleaning of the two components (in a cleanroom environment) the mould and the flat Borofloat ${ }^{\mathrm{TM}}$ glass sheet are placed in a muffle, the glass placed above the mould. The use of a muffle is necessary to ensure a better temperature distribution during the slumping process and permit also to remove the air so to avoid the convection, with obvious advantages in terms of homogeneous heat distribution, and using only the irradiation. Another advantage in using a muffle arises from the fact that it protects the mould and the glass from the dusty ambient of the oven. The muffle 
is placed inside the oven and a suitable thermal cycle will be then applied with predetermined warming-up, holding times and cooling rates. During the slumping a pressure is applied on the glass so to force it against the mould surface. This approach ensures the full contact of the glass against the mould and is also the reason for which it is necessary to manufacture the mould surface with a good microroughness. At the end of the slumping the glass sheet will have copied the shape of the mould but it will contain probably some shape errors, due to stresses arising mainly from the thermal gradients presents on its surface during the cooling-down. At that point, the proposed manufacturing process foresees the measurement of the optical shape of the segment using an astatic support and an interferometer. This support is able to remove the gravity induced deformations from the shape of the segment permitting hence to measure it in a near weightless condition. After having obtained the map of the surface, the shape errors respect to the theoretical one will be corrected using the Ion Beam Figuring technique ${ }^{(5)}$. This is a non contact figuring technique that doesn't apply on the optic any appreciable force, a necessary characteristic for these very lightweight and floppy segments. INAF-OAB has a long experience in this type of technique and has developed an Ion Beam Figuring facility that can be used for this purpose. The best possible result for the slumping technique under development will be of course the manufacturing of a segment within the optical tolerances just after the slumping. This could avoid the necessity of the Ion Beam Figuring step. Some types of errors having low spatial frequencies could be acceptable (if not having a too large amplitude) because the adaptive support hosting the segment has the capability to compensate them up to a certain degree.

\section{MOULD AND GLASS MATERIAL SELECTION}

The first stage of the slumping investigation was to define the material of the mould and the type of glass for the segments to be slumped. In pursuing this aim, a series of tests has been done and experience has been gained regarding the problems related to the slumping of glass and the search of a suitable technique able to deliver the slumped segments within the requested optical performances.

Following this purpose, it has been done an initial comparison of the theoretical thermo-mechanical parameters of suitable materials for the mould. These parameters, for the specific application of the manufacturing of a mould for slumping, can be the following:

\begin{tabular}{|l|l|l|l|l|}
\hline Mechanical & Physical & Structural & Fabrication & General \\
\hline Young's modulus & CTE & Voids, inclusions & Machinability & Availability \\
\hline Hardness & CTE homogeneity & High Temp stability & Polishability & Scalability \\
\hline & Thermal conductivity & & Optical microroughness & Mould Cost \\
\hline & Density & & Mould characterization & \\
\hline & Glass adhesion & & & \\
\hline & Transparency & & & \\
\hline
\end{tabular}

Tab. 1 Material properties

It should be noted that some of the properties listed above are temperature-dependent, a factor that must be taken into account. A consideration that must be kept in mind is that the choice of a mould material cannot be driven only by terms as simple as the thermo-mechanical material properties. The end product is the result of a process, which may affect or be affected by the substrate properties. The process control is, indeed, an integral part of the fabrication, and virtually every step, starting with considerations about the procurement of the raw material for the blank and ending with the certification of the finished component, must be considered also part of the selection process. All these considerations must be properly weighted in a merit function to reach an acceptable trade-off between the many input parameters in the effort to choose the best material for the mould.

The selection of the type of glass used for the optical segment to be slumped is closely linked to some of the characteristics of the mould itself like for example the matching of the CTEs and the range of temperatures necessaries for the thermal cycle during the slumping. The mechanical properties of the glass itself are important also for the final application of the slumped segment that will be used as a thin mirror shell for adaptive optics.

The materials considered for the mould production were Alumina, Silicon Carbide, Technical Quartz and Zerodur K20. The results of our investigation are presented in the following list with a brief description of each parameter.

* Elastic modulus: Young elastic modulus defines the deformation that the mould will experience when placed on the supports in the oven (probably 3 at $120^{\circ}$ ). In this case the best material is the Silicon Carbide that has 
stiffness about 4.7 times higher than that of the other materials. The comparison of the other three materials shows that they are not far from each other.

* Knoop Hardness: the hardness of the material is relevant for two reasons. First, the grinding, polishing and figuring of the surface is very time consuming for hard materials like Silicon carbide and Alumina. Infact this is one of the reasons why the costs of $\mathrm{SiC}$ optics are generally very high. Second, the advantage in having an hard surface is that it is very unlikely that scratches will be generated on it during the handling of the mould, the cleaning of its surface and the slumping process, when the glass sheet is placed above it and then removed after the thermal cycle

* CTE: ideally the best condition is to match the CTE of the mould with the CTE of the glass sheet to be slumped. In this way the pair (mould and glass) will expand and shrink altogether during the thermal cycle. The slumped glass will not experience a change in radius of curvature at the end of the slumping, maintaining the same radius of the mould. On the contrary, if there is a CTE mismatch between the two, the slumped glass will have in the end a change of radius of curvature that is defined by the following formula ${ }^{(6)}$ :

$$
R(\Delta T)=R_{0}(1+\alpha \cdot \Delta T)
$$

where $\Delta \mathrm{T}=\mathrm{T}_{\mathrm{g}}-\mathrm{T}_{\text {ambient }} \quad\left(\mathrm{T}_{\mathrm{g}}\right.$ is the Transformation temperature of the glass)

$\mathrm{R}_{0}=$ mould radius of curvature

$\alpha=$ CTE difference

The Silicon Carbide or the Zerodur K20 are the best choice for the mould, if it is used the Borofloat_33 glass (from Schott) that has a CTE of $3.3 \mu \mathrm{m} / \mathrm{m}^{\circ} \mathrm{K}$. This because Silicon Carbide or the Zerodur K20 have CTEs respectively of $4 \mu \mathrm{m} / \mathrm{m}^{\circ} \mathrm{K}$ and $2 \mu \mathrm{m} / \mathrm{m}^{\circ} \mathrm{K}$. The change in radius of curvature is anyway a problem that can be solved manufacturing the mould with a radius tailored to compensate the pre-computed change of radius of the segment so that the desired radius is obtained at the end of the thermal cycle.

* CTE homogeneity: the homogeneity of the CTE is important because the range of temperature applied to the mould can span up to $650{ }^{\circ} \mathrm{C}$ or more. In this scenario an inhomogeneity for example of $0.1 \mu \mathrm{m} / \mathrm{m} \cdot{ }^{\circ} \mathrm{K}$ in the material of the mould will translate in random local changes of the height of the mould of about 7 microns (mould thickness $=110 \mathrm{~mm}$ ). It is clear hence that the material of the mould should have a CTE homogeneity much more stringent than this to avoid unpredictable deformations of the surface when at $650{ }^{\circ} \mathrm{C}$. In literature, numerical data have been found for Silicon Carbide and Zerodur. The information about the Technical Quartz (Lithosil QT) specifies that the material is not certified for what concern the material homogeneity of the blank, striae and striations. The best material is surely the Zerodur K20. Since it is produced by thermal transformation of the "normal" Zerodur, that has a CTE homogeneity within $0.004 \mu \mathrm{m} / \mathrm{m} \cdot{ }^{\circ} \mathrm{K}$ for pieces up to 1.5 meters in diameter, it retains the same kind of performances in CTE constancy. With this kind of homogeneity the maximum local deviation (worst case) that can be expected is of $286 \mathrm{~nm}$ for a mould $110 \mathrm{~mm}$ thick.

* Thermal conductivity: this parameter regulates the capability of the material to quickly follow the changes in temperature during the thermal cycle. It permits to the mould to reach quickly a uniform distribution of the temperature on its surface. This is an important requirement, necessary to avoid the creation of stresses in the glass, particularly during the cooling down phase of the thermal cycle. The glass must pass very slowly through some critical temperatures (Annealing point, $\mathrm{T}_{\mathrm{g}}$, Strain point) and with the most homogeneous temperature distribution on its surface. The best material is the Silicon Carbide that has a thermal conductivity comparable to that of the metals, followed by the Alumina. The Zerodur K20 is only slightly better than the Quartz and both have poor performances in thermal conductivity when confronted with the SiC. The use of Zerodur K20 or Quartz is anyway possible if the thermal cycle employed gives time to the mould to adapt. This is possible simply increasing the time necessary to complete the cycle.

* Glass Adhesion: this parameter has been obtained from the experimental tests that have been performed during the initial phase of this study. A number of small moulds made in the four materials here considered have been used to investigate the matching between mould and glass. Using the same thermal cycle for all the moulds it has been found that the $\mathrm{SiC}$, the Alumina and the Quartz stick to the glass and hence, during the cooling phase, the mould or the glass break. Up to now the Zerodur K20 has never presented problems of sticking. The sticking can be solved using a thin coating of Boron Nitride, a well known antisticking material that can be sputtered onto the mould before the slumping. In this case it will be necessary to be sure to deposit a constant thickness of Boron Nitride to avoid changing the profile of the mould. Another material that could be used is the Platinum that can be also sputtered and resist to the temperature used in the slumping. The sputtering of the antisticking coating will add complexity to the procedure and of course it could be much better to use a material like the Zerodur K20 that, up to now, has never stuck. 
* Voids, inclusions: of course the mould should not have bubbles or inclusions because this could indicate a non homogeneous material. Alumina, $\mathrm{SiC}$ and Zerodur K20 are surely free from this problem, meanwhile, as previously already cited, the Technical Quartz is not certified. Private communications with Schott Quartz specialists have indicated that the quality of this Technical Quartz is generally much better than that indicated on the product brochure, but no certification is formally given for the homogeneity and striae.

- High temperature stability (cycles): it is important that the material has high temperature stability and does not change shape during multiple temperature cycles. All the materials here considered appear to be very stable under multiple thermal cycles.

* Max application temperature: for the slumping of glass segments it is foreseen a range of temperature up to a maximum of $700{ }^{\circ} \mathrm{C}$. Three materials have no problem on easily overcome this temperature, while the Zerodur $\mathrm{K} 20$ can reach the max application temperature of $850^{\circ} \mathrm{C}$. This anyway suits comfortably the slumping process, given that the maximum temperature foreseen is $700{ }^{\circ} \mathrm{C}$.

* Machinability: this parameter refers to the easiness of manufacturing of the mould from the mechanical point of view. The difficulty to bring the shape of the blank to the final dimension of the mould depends on the hardness of the material. For Alumina and SiC this step is generally done when the raw material has been isostatically pressed, the so called "green body" phase. In this state the material can be easily machined and shaped near to the final dimensions. Afterward the piece is fired to high temperature (sintered) and became very hard and difficult to work. In this phase there is also an isotropic dimensional shrinkage of the material up to $20 \%$. The Quartz is a very well known material that poses no difficulties during the manufacturing. The Zerodur $\mathrm{K} 20$ is obtained from the transformation of the classical Zerodur. The mould can be manufactured completely in Zerodur (that is a softer material than the K20) and then the piece is thermally transformed in Zerodur K20 maintaining essentially the same dimensions. At this point, like for Alumina and $\mathrm{SiC}$, the optical surface of the mould is polished and figured.

* Polishability and figuring: since Alumina and $\mathrm{SiC}$ are very hard materials it is no surprising that the polishing and figuring for both must use specific grinding materials and it is also very time consuming. If compared to these materials the Quartz is quite easily figured. The Zerodur K20 is harder than the Quartz but well within the normal polishing and figuring techniques used in optics.

* Microroughness: this parameter indicates the limit at witch is it possible to polish a surface made in that material. The $\mathrm{SiC}$ and Quartz can reach a microroughness of less than $5 \AA \mathrm{rms}$ meanwhile the Alumina is slightly higher. The Zerodur K20 can be polished down to $10 \AA$ A. Since the slumping will be done in direct mode (optical surface of the glass segment placed in contact with the mould surface) the microroughness of the mould is potentially important. Infact, during the slumping tests performed it has been noted that it is possible to reach a partial copy capability of the microroughness of the mould. This fact implies a full contact between glass and mould, without sticking. Hence it is probably important to be able to polish adequately the surface of the mould if a very high copy capability is requested.

* Transparency and Mould characterization: the transparency of the material is important in particular during the manufacturing of the convex mould and is related to its characterization and certification. The Quartz is the only transparent material.

The procedures foresee for the characterization and certification of the mould are:

$>\quad$ With a transparent material it is possible to create on the bottom of the mould a transparent concave surface (the mould should have a concave back surface anyway to have a constant thickness for thermal reasons) that can be easily figured and measured interferometrically. Then, looking through the material from the bottom and assuming a constant index of refraction, the upper surface will appear concave too. In this way it will be possible in principle also measure interferometrically its shape to determine the errors during the figuring and the certification process.

$>\quad$ With an opaque material like Zerodur K20 or others, the upper procedure is no longer possible. Another approach foresees the use of a 3D machine (with an accuracy of 1 microns or better) to asses the overall geometry of the mould on the longer spatial frequencies where the tolerances rms are larger. To asses the shorter spatial frequencies will be manufactured a concave spherical master having the same radius of curvature of the mould and with a diameter of about $25-30 \mathrm{~cm}$. This will be used to check patches of the mould for high spatial frequencies.

* Material availability: the Alumina, Silicon Carbide and Quartz are materials that are available from a number of suppliers. For Alumina and $\mathrm{SiC}$ the maximum blank sizes available are near to the requirement of this study 
(mould of $0.7 \mathrm{~m}$ diameter for slumping of segments of $0.5 \mathrm{~m}$ diameter). The Quartz and Zerodur K20 can be also produced in blanks of this size. In the case of the Zerodur K20 the only supplier is Schott.

* Scalability to $1.5 \mathrm{~m}$ : the issue of the scalability foresees the possibility to scale up to 1.5 meter the slumping process. This size exceeds the maximum diameters available for blanks in Alumina and Silicon Carbide. In this case to produce a mould it will be necessary to braze a number of smaller petals. This process has been used to manufacturing large $\mathrm{SiC}$ mirrors for space missions. Its use, for the manufacturing of a mould that must withstand to high temperatures, should be studied and a number of problems could arise, for example if the CTE of the brazing material is not equal to that of the petals. The cost of this procedure is also an issue. The Technical Quartz can be produced in blanks up to 1.5 meters meanwhile the Zerodur K20 can be produced with dimensions of several meters.

* Mould cost: the most expensive mould is the one made in Silicon Carbide, while the manufacture of a mould in one of the other three materials is less expensive. Respect to Silicon Carbide, the mould in Alumina or Technical Quartz will cost about 40\% less, the mould in Zerodur K20 about 30\% less.

Using the considerations discussed it was decided that a good trade-off for the material of the mould is the Zerodur K20 $0^{(7)}$ paired with the Borofloat_33 glass for the mirror shell. This decision is supported from the following main considerations (but not only):

D The Zerodur K20 has a CTE near to the Borofloat_33, the Schott glass that will be used for the slumped segments.

$>$ The CTE homogeneity of the Zerodur K20 is the highest of all the other materials. This is an important parameter.

$>\quad$ The Zerodur K20 has not shown sticking attitude to the glass within the thermal cycles at present (up to 670 ${ }^{\circ} \mathrm{C}$ ) and hence doesn't need any anti-sticking coating. The sticking of the glass on the mould is an event that very likely can create a heavy damage to the mould, requiring a re-figuring.

$>$ The dimensions of Zerodur K20 blank can be easily scaled-up to 1.5 meters in diameter.

$>$ The characterization of a non transparent mould using a 3D machine for the measurement of the geometry (long spatial frequencies) and of a $25-30 \mathrm{~cm}$ spherical master to control the higher spatial frequencies on patches of the mould is a well known and trusted technique.

$>$ The cost of the finished mould in Zerodur K20 is not far from that of the other moulds made in the other materials (except for the Silicon Carbide).

\section{OVERVIEW OF THE IMPROVEMENTS OBTAINED IN THE SLUMPING TECHNIQUE}

In the following pages will be shown some of the most representatives steps performed during the development of the slumping technique. A facility, that comprises two ovens of different sizes, has been setup for this purpose.

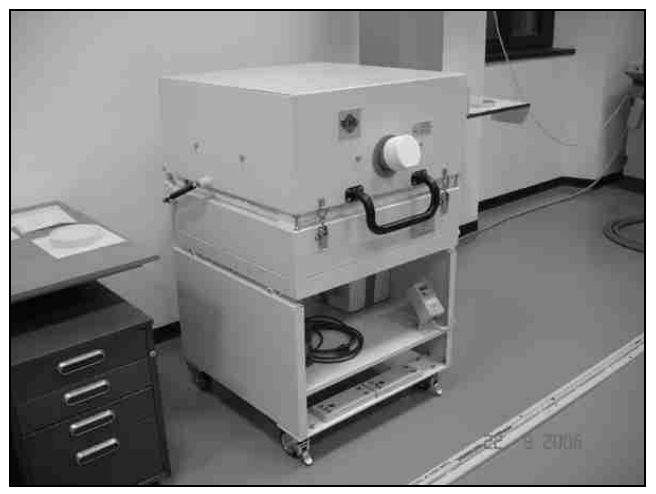

Fig. 3 Oven for the tests

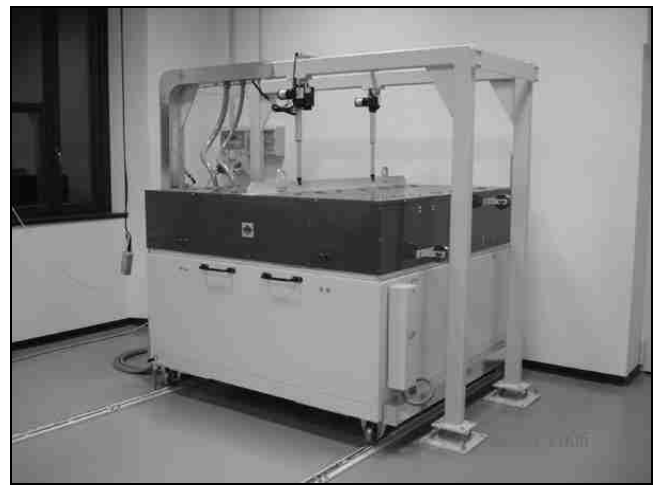

Fig. 4 Full size oven

The slumping tests described in this paper have been performed in the smaller one, visible in Fig. 3. The larger oven, visible in Fig. 4, has been manufactured $a d-h o c$ to permits a fine control of the temperature in all the internal oven volume and will be used for the slumping of the full size thin shell segments. Its capability can permit the slumping of 
segments up to 1.1 meters in diameter. The slumping tests were made on rectangular $(100 x 50 \mathrm{~mm})$ and circular $(\varnothing 130$ $\mathrm{mm}$ ) samples Borofloat 33 glass sheets having thickness of $2 \mathrm{~mm}$. A small Zerodur K20 mould was used for the tests, having a diameter of $150 \mathrm{~mm}$. The two surfaces were grinded and figured to obtain on one side a spherical convex surface with radius of curvature $3950 \mathrm{~mm}$ (sag about $0.71 \mathrm{~mm}$ ) and on the other side a flat surface (sag about $6 \mu \mathrm{m}$, concave). The microroughness of both the mould surfaces was of about $5 \mathrm{~nm}$ rms.

In Fig. 5 is shown the result of a slumping performed on the convex side of the mould using a circular sample. The experiment was performed applying a uniform pressure of about $16 \mathrm{gr} / \mathrm{cm}^{2}$ on the glass during the holding time of the thermal cycle, when the glass was in a plastic state. To protect the mould and glass from the dust of the oven a metallic cover was placed above the mould during the thermal cycle. In the case of a good slumped segment, after the coolingdown, we expected to see a circular pattern of interference fringes due to the change of radius of curvature of the glass respect to the mould. The mould and glass were illuminated with Sodium light $(\lambda=590 \mathrm{~nm})$ to show the interference fringes due to the air gap present between the mould and glass surfaces. Even if the fringes were visible, there were patches of glass that showed no fringes. Some of the patches were due to dust specks but others were simply areas where the glass was not pushed in full contact with the mould. The problem was due to the not complete homogeneous behavior of the pressure applied that in some areas was unable to push completely against the glass disc, instead discharging the pressure on the surrounding areas. The proof of concept was anyway correct, demonstrating that the application of a homogeneous pressure could be a viable technique to be pursued for the slumping of thin glass shells.

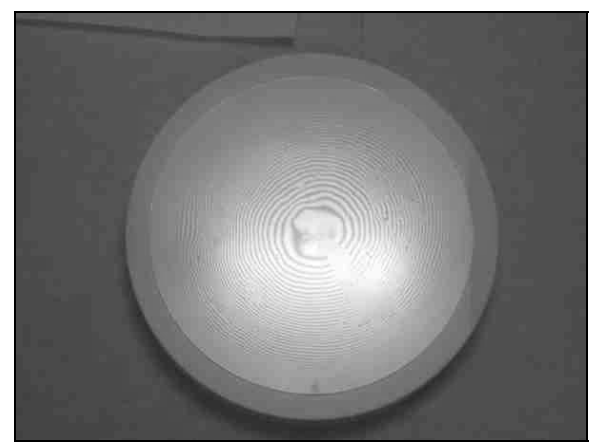

Fig. 5 Hot slumping with pressure of about $16 \mathrm{gr} / \mathrm{cm}^{2}$

In another experiment the flat side of the mould was used to investigate the spatial frequencies copy capability of the slumping process. A rectangular segment was slumped and then the spatial frequencies present on its surface were compared against those present on the mould itself. In Fig. 6 are visible the fringes due to the shape difference between segment and mould before the slumping. The experiment was carried out in same conditions as previously described: hot slumping with a pressure of about $16 \mathrm{~g} / \mathrm{cm}^{2}$. When the weight was removed and the slumped segment, placed above the mould, was exposed to the Sodium light, a large area of the segment was in contact with the mould. Very few fringes were present and also almost no dust specks were visible. The segment was so in contact that apparently it had stuck against the mould. This was not true but the effect was due only to the optical contact between the two surfaces. In Fig. 7 is shown the segment just after the removal of the weight. It must be noted that the wavelength of the Sodium light is 590 $\mathrm{nm}$ and that the difference in height between two dark fringes in this picture is of $295 \mathrm{~nm}$. As visible, a large part of the area of the segment was in very good contact, having only a difference in height of $295 \mathrm{~nm}$. After several minutes the air started to enter slowly between the two surfaces and the pattern of fringes begins to modify. At the end of the process, a system of circular fringes was visible (Fig. 8). For this experiment the segment was not centered on the mould but located laterally, near to its edge. Even if the segment was out of axis respect to the mould, the fringes were almost centered along the axis of the mould. Infact, this effect is due to the radial difference in temperature of the mould during the cooling-down. This inhomogeneity in the temperature distribution was observed in the mould during a previous characterization using thermocouples. It can be controlled or minimized with a more accurate tuning of the thermal cycle parameters.

As already cited in this paper the difference in CTE between mould and glass generates a change of radius of curvature. In this case the two surfaces were essentially flat with a very long radius of curvature and the two CTEs are not very far from each other. A computation of the number of fringes that should be visible in this situation revealed that less than one fringe should be attributable to this CTE effect. The 12 fringes instead visible are generated from the combination of 
essentially two other effects: the radial difference in temperature between center and edge of the mould and the difference in temperature between the upper and lower surface of the glass segment during the cooling-down. Both these effects introduce stresses in the glass during the cooling-down because they pass through critical temperatures in a non homogeneous way.

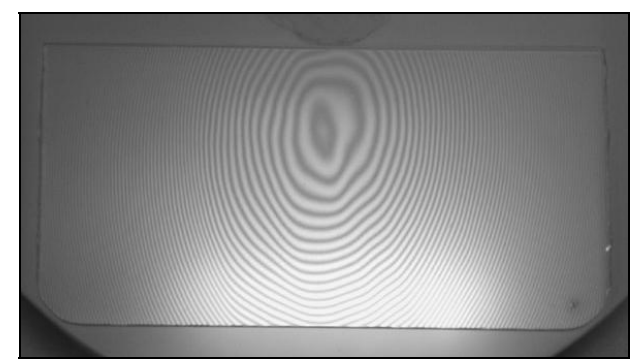

Fig. 6 Segment before the slumping

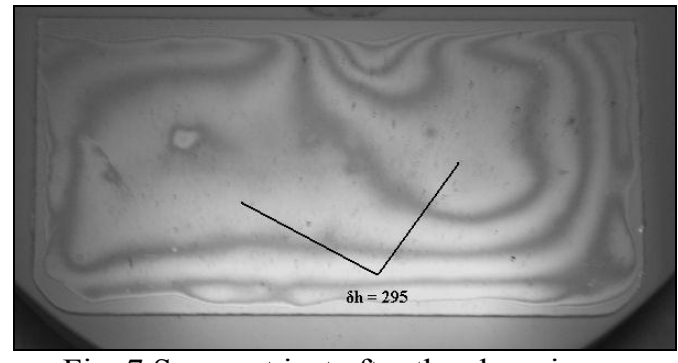

Fig. 7 Segment just after the slumping

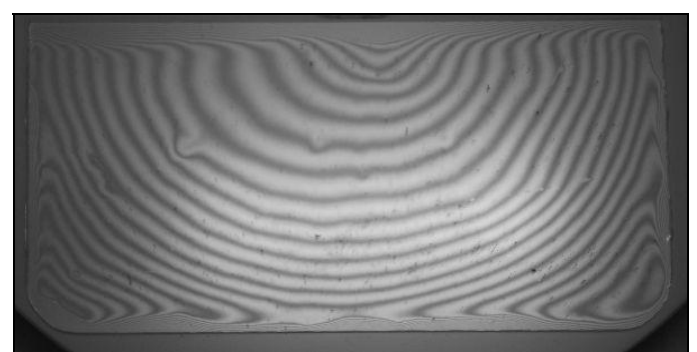

Fig. 8 Pattern of fringes after several minutes

During the cooling-down phase of a thermal cycle, the upper surface of the glass is a fraction of a degree Celsius warmer or cooler (depending from the setup and materials surrounding and in contact with the glass). When the glass passes through the Transition Temperature (Tg), at which the material start to behave like a solid, the passage happens in different times and this introduces strain between the upper and lower surface that is translated in a change of shape of the glass. The formula ${ }^{(6)}$ that quantifies the change of focal length due to this effect is:

where:

$$
\delta F=-2 \cdot\left(\frac{F^{2}}{S}\right) \cdot \alpha_{t} \cdot\left(T_{b a c k}-T_{\text {front }}\right)
$$

$$
\begin{array}{ll}
\delta \mathrm{F}=\text { change of focal length } & \mathrm{F}=\text { focal length } \\
\mathrm{S}=\text { glass thickness } & \alpha_{\mathrm{t}}=\mathrm{CTE} \\
\mathrm{T}_{\text {back }}=\text { back surface temperature } & \mathrm{T}_{\text {front }}=\text { front surface temperature }
\end{array}
$$

In this experiment the difference in temperature between the upper and lower surface of the sample was not known during the cooling-down. Anyway, in literature ${ }^{(6)}$ there are reports that even only a difference of $0.2^{\circ} \mathrm{C}$ between the two faces of a $1 \mathrm{~mm}$ thick glass piece having $6 \mathrm{~m}$ focal length and CTE $7.5^{*} 10^{-6}$ can generate a change of focal length of 108 $\mathrm{mm}$. To control this effect it is important to reduce the temperature difference between the glass thickness for example using very low cooling down phases in the thermal cycle, during the critical temperatures. It must be noted anyway that, in the worst case in which this effect will not be totally removed, there can be again a change of focal and hence a change of radius of curvature (if the difference in temperature between the faces is constant in every point of the glass). This in principle could be corrected manufacturing a mould with a radius tailored to compensate the effect.

In Fig. 9 are shown a number of profiles of the segment before the slumping, taken with the LTP (Long Trace Profilometer) available in INAF-OAB. In Fig. 10 instead are shown the profiles of the segment after the slumping. As can be seen, the shape passed from concave to convex, as expected, because the flat side of the mould was concave with a max sag of 6 microns (about $400 \mathrm{~m}$ of radius). The P-V of the slumped segment was only of 2.5 microns and not 6 , in agreement with the number of fringes visible in Fig. 8. Infact, these 12 fringes, that indicate the shape difference between mould and glass, shows a sag of about 3.5 microns. 


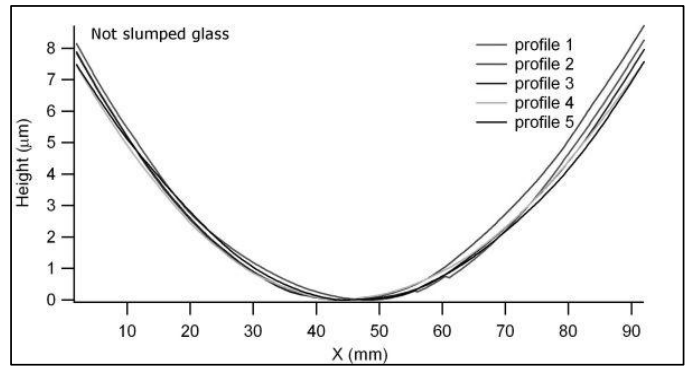

Fig. 9 Segment profiles before the slumping

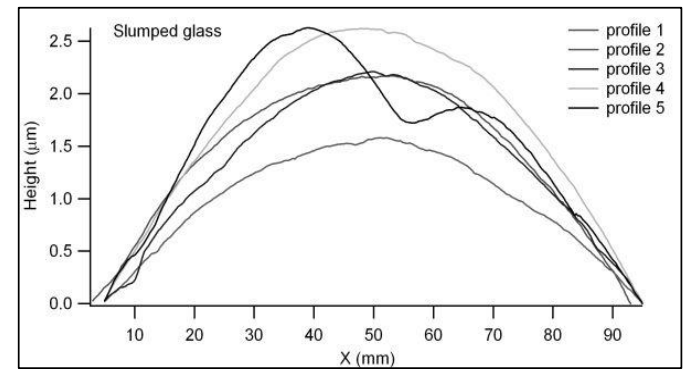

Fig.10 Segment profiles after the slumping

Fig. 11 shows the PSDs of the segment before and after the slumping and the PSD of the mould (obtained with the LTP profilometer and Zygo interferometer). The data of the slumped glass are contained within the PSD curves of the nonslumped segment and of the mould. This means that the smoothness of the slumped glass was improved by the slumping process in the spectral range from about $1 \mathrm{~mm}$ to $30 \mathrm{~mm}$. It must be remembered that this was a hot pressure slumping made at $16 \mathrm{gr} / \mathrm{cm}^{2}$.

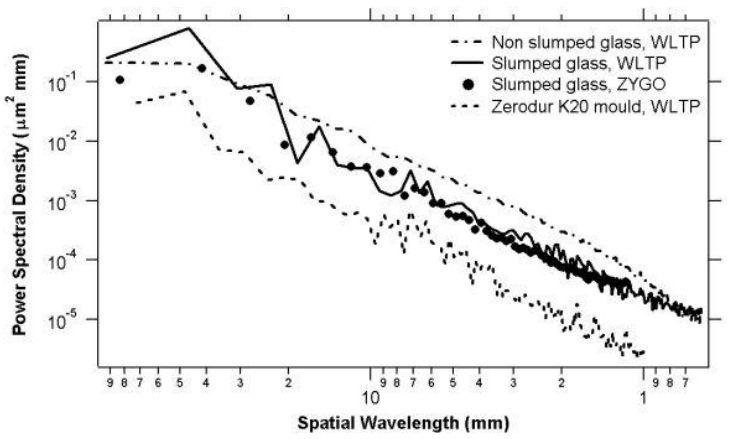

Fig.11 PSDs before and after the slumping

In another test, to check the capability of the slumping process to copy the very high spatial frequencies of the mould, a flat sample was pressed against the mould flat surface with a pressure of $60 \mathrm{gr} / \mathrm{cm}^{2}$. A very low cooling rate was also used of $0.5^{\circ} \mathrm{C} / \mathrm{min}$. At the end of the thermal cycle the segment showed a large area in full contact with the mould (Fig. 12). After the removal of the segment a comparison of the segment microroughness with the microroughness of the mould was performed. The copy capability of the glass at this pressure is well shown in Fig. 13, as seen through a Nomarski microscope. A scratch was present on the flat side of the mould used in this experiment. This scratch was about $40 \mathrm{~mm}$ long and crossed by another lighter scratch. These features were used as reference points to find the same points on the glass surface. The features were fully copied by the glass. The picture shows the scratch on the mould and on the glass. Clearly the features were fully copied (the unfocused pattern on the sample is on the back side of the glass). A spatial frequency analysis was performed to show the copy performance and is shown in Fig. 14. This graph shows that the microroughness of the sample match quite well that of the mould.

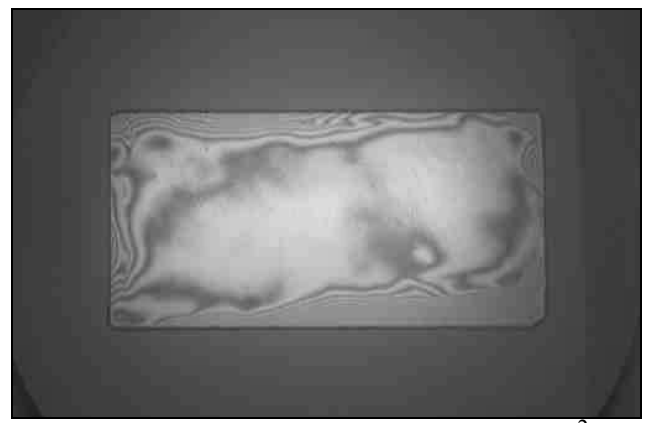

Fig.12 Segment slumped at $60 \mathrm{gr} / \mathrm{cm}^{2}$ 


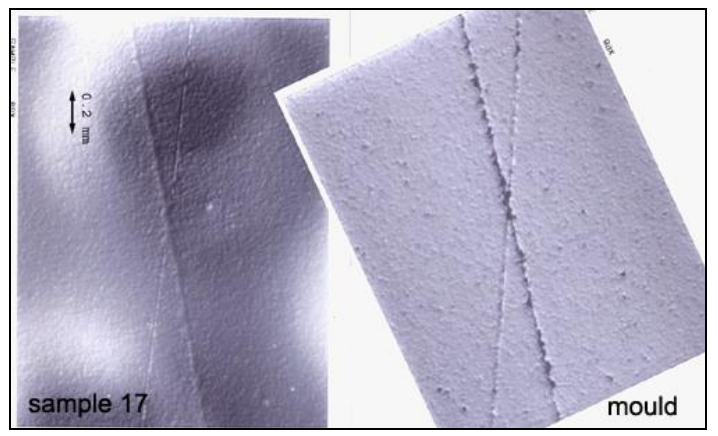

Fig.13 Nomarski photograph

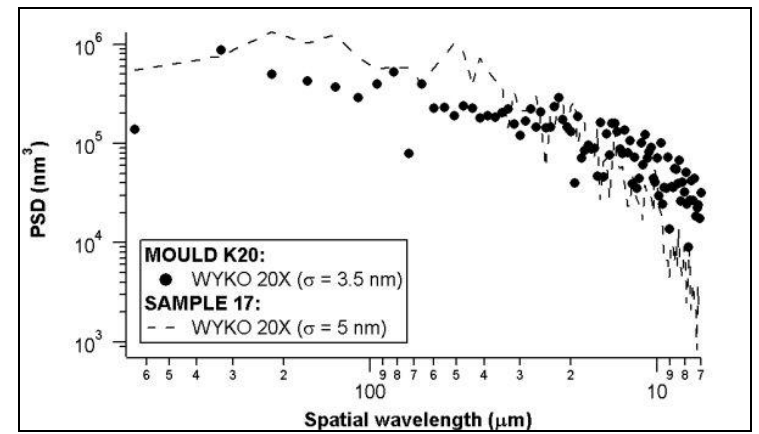

Fig. 14 Mould-Segment PSD comparison

\section{PRESENT STATUS OF THE INVESTIGATION}

After having developed a suitable procedure and technology for the slumping of thin glass shells we have started to slump methodically a number of circular Borofloat_33 disks having diameters of $130 \mathrm{~mm}$. This phase is nowadays under development and concern the individuation and refinement of the best suitable thermal cycle. At this point of the development the dust is no longer a problem and we have the capability to obtain full contact on all the surface of the glass against the mould and to control the amount of pressure applied, as visible for example in Fig. 15 where is shown a square sample of slumped glass. In Fig. 16 is instead shown the $150 \mathrm{~mm}$ diam. K20 mould used for the slumping tests. These tests have been done using a vacuum muffle made in stainless steel. The use of a muffle offers many advantages because it protects the glass and mould from the ambient of the oven. It spreads evenly the heat reducing the thermal inhomogeneity intrinsic of the oven that has electric resistances located in well defined places. It permits also to remove the inner air and to use only the irradiation for the heating, avoiding the convection that creates a thermal gradient. The convection could also potentially bring around the dust particles that are anyway present on the inner walls of the muffle and the vacuum help to keep at bay this potential problem.

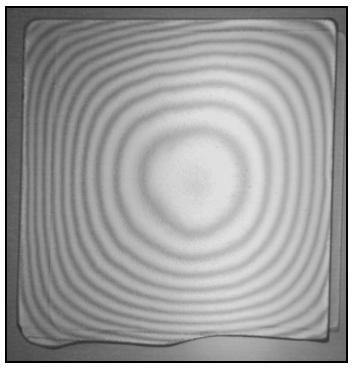

Fig.15 Fringes very sharp up to the edge and no dust

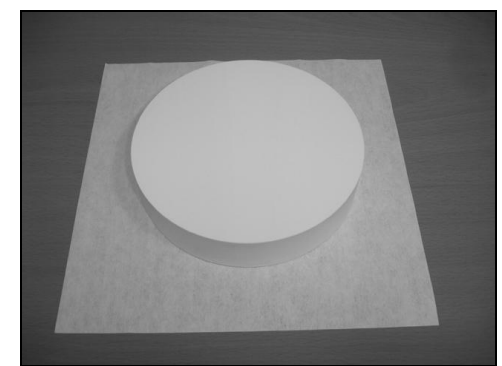

Fig.16 Zerodur K20 mould (150 mm diam.)

The results obtained so far with the slumping of $130 \mathrm{~mm}$ circular disks has been obtained changing the temperatures and times of the thermal cycles employed, in an effort to find the best combination of warming-up, holding time and coolingdown times. For this reason the results obtained differs each other of some amount. The investigation on the repeatability of the slumping process will be performed in the future, when it will be selected a particular thermal cycle. The measurements of the shape of the slumped spherical segments have been done by means of a Zygo Interferometer. The mould shape was measured with a 3D machine to obtain the radius of curvature and with a Wyko profilometer for the assessment of the microroughness. Its optical shape has not yet been measured interferometrically because it has a convex shape that it is notoriously difficult to measure. We are preparing an interferometric setup to be able to obtain this critical information that will be then compared with the shapes of the segments slumped. In this situation we can only compare between them the concave slumped segments. In all the four segments till now slumped it is visible a pattern of features that repeat itself with a good approximation. In Fig. 17 and Fig. 18 are shown for example the segments M1 and M4 measured on a diameter of $80 \mathrm{~mm}$. It is clearly visible that on their surface are present very similar features. The same features are present also on the other two segments not shown. It is very likely hence that these features are present on the mould and are simply replicated onto the segment surface. This is an indication that the process has a very good copy capability. The optical shape of the segments M1 and M4 was also very good at $80 \mathrm{~mm}$ diameter, showing respectively a figure error of $70 \mathrm{~nm}$ rms $(\lambda / 9)$ and $57 \mathrm{~nm}$ rms $(\lambda / 11) @ 632.8 \mathrm{~nm}$. 


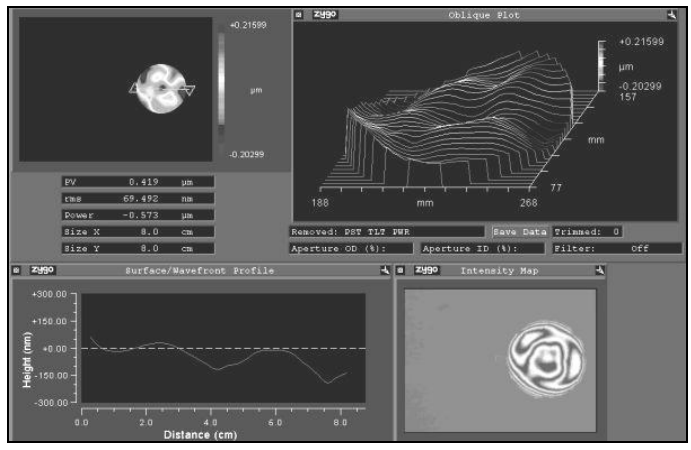

Fig. $17 \mathrm{M} 1$ slumping on $80 \mathrm{~mm}(\lambda / 9)$

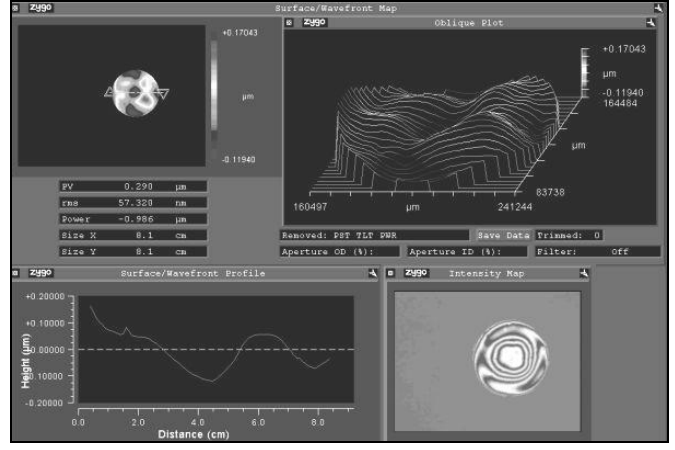

Fig. $18 \mathrm{M} 4$ slumping on $80 \mathrm{~mm}(\lambda / 11)$

In Fig. 19 is instead shown the overall shape of the segment M4 on $130 \mathrm{~mm}$ diameter. In this case the optical shape of the segment has an error of about $218 \mathrm{~nm}$ rms $(\lambda / 2.9)$. This measure very likely resembles the optical figure of the mould that was polished without addressing precisely its spherical shape because it was used to investigate the preliminary part of this study on the slumping. In this sense the mould is presently limiting the optical performances obtainable from the slumping of the segments. In Fig. 20 are shown the fringes that were present on one of the slumped segments when placed on the mould under Sodium light. As visible the fringes shows a remarkable circularity and their number is much lower than that of a very initial test shown in Fig. 5. This indicates that, in this last case, the contact with the mould during the slumping was much more complete, and the thermal stresses introduced by the thermal gradients were also minimized, due to the improvements obtained in the slumping process.

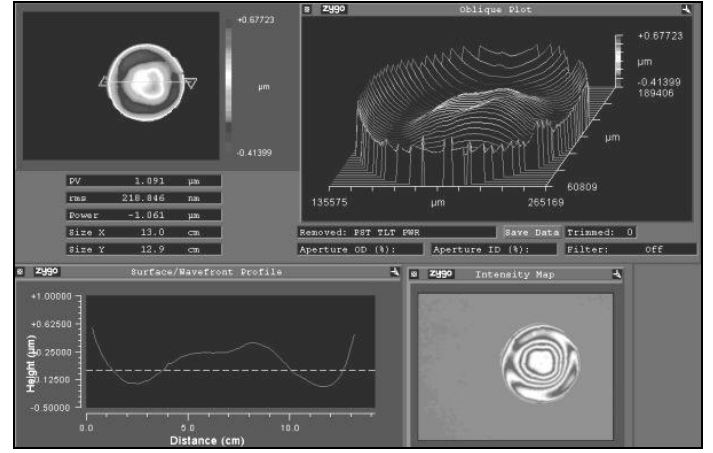

Fig. 19 M4 slumping on $130 \mathrm{~mm}(\lambda / 2.9)$

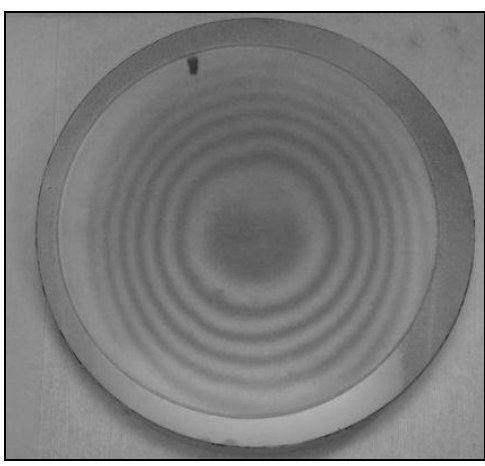

Fig. 20 Segment on the mould

In Table 2 are shown the results obtained on all the four segments till here slumped. The goal requested for the optical surface quality of the final $500 \mathrm{~mm}$ prototype ${ }^{(8)}$ up to a diameter of $140 \mathrm{~mm}$ is also shown under the "Specification" column. As previously said the $150 \mathrm{~mm}$ mould is limiting the performances obtainable with this slumping technique, and it has at the present an optical figure that is surely not in specs as requested for a real segment. Definitive results will be obtained comparing the shape of the slumped segments with the optical map of the mould, a task that will be performed in the next weeks. Further, in the next few months we will start to slump using the large mould (in specs) able to produce the $500 \mathrm{~mm}$ diam segments using also a large vacuum muffle for the manufacturing.

\begin{tabular}{|c|l|l|l|l|l|}
\hline $\begin{array}{c}\text { Spatial wavelength range } \\
{[\mathbf{m m}]}\end{array}$ & \multicolumn{1}{|c|}{ Specification } & \multicolumn{1}{|c|}{ TEST 1 } & \multicolumn{1}{c|}{ TEST 2 } & \multicolumn{1}{c|}{ TEST 3 } & \multicolumn{1}{c|}{ TEST 4 } \\
\hline & & & & & \\
\hline $0-60$ & $\lambda / 33$ & $\lambda / 13$ & $\lambda / 11$ & $\lambda / 9.7$ & $\lambda / 12.6$ \\
\hline $0-80$ & $\lambda / 31$ & $\lambda / 9$ & $\lambda / 6$ & $\lambda / 6.4$ & $\lambda / 11$ \\
\hline $0-100$ & $\lambda / 26$ & $\lambda / 4.8$ & $\lambda / 3$ & $\lambda / 4$ & $\lambda / 7.2$ \\
\hline $0-120$ & $\lambda / 18$ & $\lambda / 2.8$ & $\lambda / 1.8$ & $\lambda / 2.7$ & $\lambda / 3.8$ \\
\hline $0-140$ & $\lambda / 12$ & $\lambda / 2.4$ & $\lambda / 1.7$ & $\lambda / 2.2$ & $\lambda / 2.9$ \\
\hline
\end{tabular}

Tab. 2 Slumping results on $130 \mathrm{~mm}$ diam. segments 


\section{CONCLUSIONS}

The conclusions that can be drawn after this part of study on the slumping technique indicate that the procedure developed has the potential to reach a very high shape accuracy on the slumped segments. The material that has been chosen for the mould (the Zerodur K20 from Schott) has no tendency to stick to the Borofloat_33 at the temperatures used for these slumping and up to $670{ }^{\circ} \mathrm{C}$. This permits to use directly its surface for the slumping, without the need of an antisticking layer. Further, it has been determined that the use of pressure to force the glass in full contact with the mould is necessary at the temperature used in these slumping tests. Without pressure the time necessary for obtaining a good contact between the surfaces can be very long and in some cases also impossible to obtain. With this approach the mould microroughness should be of 2-3 nm rms or less because the Borofloat_33 will copy either the mould microroughness and its shape at lower spatial frequencies. The use of a muffle is surely an important factor of this technique for a number of advantages that permit to obtain. The slumping technique developed very likely has not yet reached its limits in terms of performances. The fact that the shape of the small mould used in the tests is not well known doesn't permit a direct comparison with the slumped segments but this data will be obtained in the next few weeks because an interferometric setup able to measure its convex shape is under construction. This mould was made for the initial tests and at that time it was not considered necessary to know in detail its shape, considering also the difficulty to measure a convex shape. Anyway, it's clear from the measurements of the $130 \mathrm{~mm}$ glass disks already slumped that there is a repeated pattern on their surfaces, indicating that the opposite of this pattern is very likely also present on the mould. This means that the process is able to deliver a good copy capability and that the results till here obtained are limited from the quality of the mould optical surface and not from the slumped segments, that limit themselves to copy its surface. The larger mould under construction for the manufacturing of the $500 \mathrm{~mm}$ diam slumped segment will be well characterized in terms of optical shape and will have tight specifications. In the next few months it will be delivered not only this large mould but also the larger muffle to be used in the slumping of a number of $500 \mathrm{~mm}$ full size segments that will bring to the production of a useful adaptive mirror that is the deliverable of this study.

\section{REFERENCES}

1. J. Hill and P. Salinari, "Large Binocular Telescope Project", Proc. SPIE 4837, 2002

2. A. Riccardi, G.Brusa, P. Salinari, D. Gallieni, R. Biasi, M. Andrighettoni, H. Martin, "Adaptive secondary mirrors for the Large Binocular Telescope", Proc. SPIE 4839, 2003

3. F. Wildi, G. Brusa, A. Riccardi, M. Lloyd-Hart, H. Martin, L. Close, "Towards first light of the 6.5m MMT adaptive optics system with deformable secondary”, Proc. SPIE 4839, 2003

4. M. Ghigo, S. Basso, O. Citterio, F. Mazzoleni, D. Vernani, "Manufacturing of lightweight glass segments for adaptive optics", Proc. SPIE 6272, 2006

5. M. Ghigo, P. Conconi, E. Antonello, G. Pareschi, "Accurate ion beam figuring of field correctors for optical and UV space instrumentation", Proc. OPTRO 2005 International Symposium, (2005)

6. R.N.Wilson, "Reflecting Telescope Optics II", Astronomy \& Astrophysics Library, ISBN 3-540-60356-5, SpringerVerlag, Pag. 217,218

7. Thorsen Doring, Ralf Jedamzik, Peter Hartmann, Hauke Esemann, Clemens Kunisch, "Forming mandrels for X-ray telescopes made of modified Zerodur", 2004, Proc. SPIE v.5168, p. 148

8. A. Riccardi, "Optical figuring specifications for thin shells to be used in adaptive telescope mirrors," in Advances in Adaptive Optics II, B. L. Ellerbroek and D. Bonaccini Calia, eds., vol. 6272 of Proc. SPIE, p. 50, July 2006

\section{ACKNOWLEDGEMENTS}

This study has been financed by the European Community in the frame of OPTICON FP6 for the ELT Design Study (WP9300) contract $\mathrm{n}^{\circ} 011863$ 